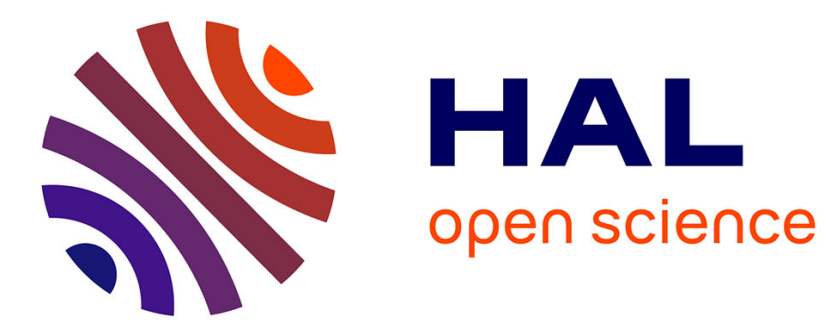

\title{
Low-Complexity Approximations for LMMSE Channel Estimation in OFDM/OQAM
}

\author{
Vincent Savaux, Yves Louët, Faouzi Bader
}

\section{To cite this version:}

Vincent Savaux, Yves Louët, Faouzi Bader. Low-Complexity Approximations for LMMSE Channel Estimation in OFDM/OQAM. 2016 23rd International Conference on Telecommunications (ICT), May 2016, Thessaloniki, Greece. 10.1109/ict.2016.7500464 . hal-01311058

\section{HAL Id: hal-01311058 \\ https://hal-centralesupelec.archives-ouvertes.fr/hal-01311058}

Submitted on 1 Jun 2016

HAL is a multi-disciplinary open access archive for the deposit and dissemination of scientific research documents, whether they are published or not. The documents may come from teaching and research institutions in France or abroad, or from public or private research centers.
L'archive ouverte pluridisciplinaire HAL, est destinée au dépôt et à la diffusion de documents scientifiques de niveau recherche, publiés ou non, émanant des établissements d'enseignement et de recherche français ou étrangers, des laboratoires publics ou privés. 


\title{
Low-Complexity Approximations for LMMSE Channel Estimation in OFDM/OQAM
}

\author{
*Vincent Savaux, **Yves Louët, and **Faouzi Bader \\ **SCEE/IETR - CentraleSupélec, Rennes. \\ Avenue de la Boulaie, 35576 Cesson - Sévigné Cedex, France. \\ Emails: yves.louet@centralesupelec.fr, and faouzi.bader@supelec.fr
}

\begin{abstract}
In this paper, the authors describe and compare two low-complexity approximations of the linear minimum mean square error (LMMSE) channel estimation method for orthogonal frequency division multiplexing/offset quadrature amplitude modulation (OFDM/OQAM) systems. Simulations reveal that we are able by proposed approximations to reduce the complexity of the LMMSE estimator without degrading the overall BER system performance.
\end{abstract}

Keywords-OFDM/OQAM, Channel estimation, MMSE, Approximation methods.

\section{INTRODUCTION}

Among the alternatives to orthogonal frequency division multplexing (OFDM), OFDM/Offset quadrature amplitude modulation (OFDM/OQAM) is a potential candidate for the fifth generation $(5 \mathrm{G})$ wireless systems [1]. In fact, this modulation scheme is more flexible, more spectrally efficient, and generates less out-of-band interferences than OFDM. In order to obtain these good features, the constraint of complex orthogonality among symbols and subcarriers is relaxed, hence the used prototype filters are designed in order to minimize the out-of-band interferences [1], [2]. However, the used pulse shapes overlap in both time and frequency, therefore they generate inherent interferences among symbols and subcarriers such as illustrated in Figs. 1-(b) and 2, where $m$ and $n$ correspond to the frequency and time indexes, respectively.

The presence of these distortions makes channel estimation more complicated in OFDM/OQAM than in OFDM systems. Overviews on different preamble-based channel estimation methods in OFDM/OQAM have been deeply presented in [3]-[5]. More recently, an adaptation of the linear minimum mean square error (LMMSE) technique using OFDM/OQAM modulation scheme has been proposed in [6]. It has been observed in [6] that, as in OFDM systems, the complexity of the LMMSE estimator is one of its main drawback.

The linear minimum mean square error channel estimation method presented by Edfors et al. in [7] for OFDM modulation scheme is in this paper proposed, adapted, and implemented for OFDM/OQAM modulation. In OFDM the LMMSE in [8], allows the estimator to smooth the noisy channel frequency response estimated with the Least Square (LS) criterion. In OFDM/OQAM, the intrinsic interference

\footnotetext{
*CentraleSupélec is the former institution of Vincent Savaux, actually with $\mathrm{b}<>$ com research institute at:vincent.savaux@b-com.com.
}

is an additional source of errors in the channel estimation process [5], [9]. As a consequence, using LMMSE as a spectral smoothing filter appears to be very relevant for the considered filter bank modulation scheme [10].

In this paper, we present two approximations of LMMSE allowing to reduce the complexity of this estimation method. The first approximation is based on the low-rank approximation (LRA)technique originally used in OFDM [7], the principle of which is to replace the unknown exact channel covariance by an approximated one. The second approximation is based on the fact that the noise covariance matrix is cyclic in OFDM/OQAM due to the receiver structure, and by using the diagonalization basis of the approximated channel covariance matrix used in the LRA method is then replaced by the Fourier basis, which allows us to reduce the complexity of LMMSE from $\mathcal{O}\left(M^{3}\right)$ to $\mathcal{O}\left(M \log _{2}(M)\right)$ scalar multiplications. Simulations results show that both proposed approximations do not reduce the performance of LMMSE (in terms of bit error rate (BER)) from $E_{b} / N_{0}=0 \mathrm{~dB}$ to $25 \mathrm{~dB}$.

The rest of the paper is organized as follows: The system model is introduced in Section II, and the two simplified LMMSE methods are presented in Section III. The simulations are provided in Section IV, and we finally draw conclusions in Section V.

\section{System Model}

The transmission of OFDM/OQAM symbols over a frequency selective channel is considered with a perfect time and frequency synchronization. The general structure of an OFDM/OQAM modem is depicted in Fig. 1-(a). The transmitter contains a synthesis filter bank (SFB) and the receiver is composed of an analysis filter bank (AFB). The structure in Fig. 1-(a) highlight that both SFB and AFB consist of two steps: i) a fast Fourier transform (FFT) or its inverse (IFFT); ii) a polyphase network (denoted by PPN in Fig. 1-(a)) consisting in a set of digital filters which are described afterward.

At the frequency-time position $(m, n)$, the output of the AFB is 


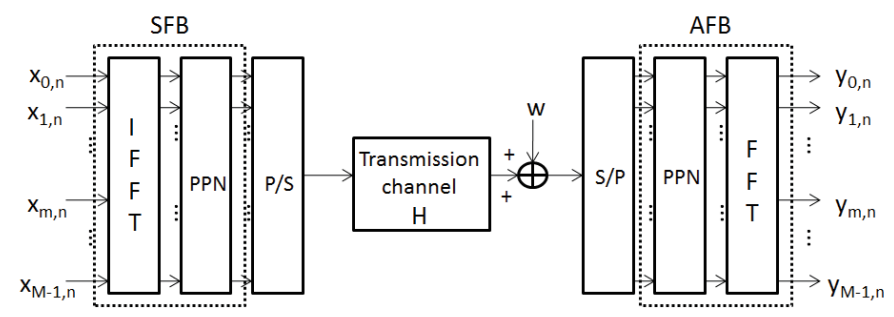

(a) Polyphase implementation of the OFDM/OQAM modem.

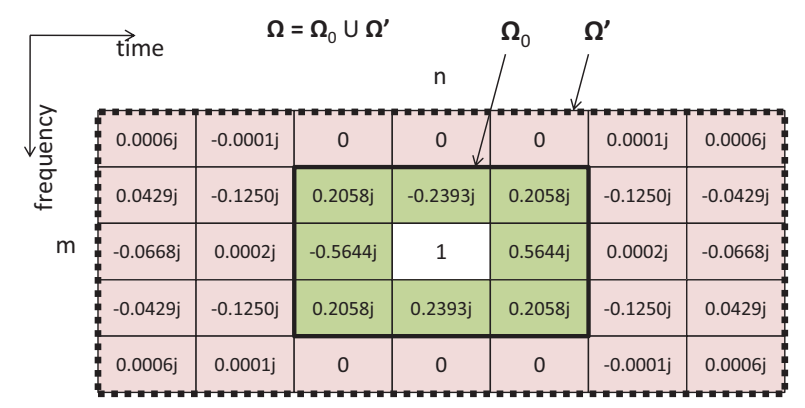

(b) Transmultiplexer response of the OFDM/OQAM system using Bellanger's filter [11].

Fig. 1. OFDM/OQAM transmultiplexer, (a) Polyphase implementation of the OFDM/OQAM modem, (b) Transmultiplexer response.

$$
y_{m, n}=H_{m, n} x_{m, n}+\underbrace{j \sum_{(p, q) \in \Omega} H_{p, q} x_{p, q}<g>_{p, q}^{m, n}}_{I_{m, n}}+w_{m, n},
$$

where $x_{m, n}$ is the real OQAM symbol transmitted at frequency-time position $(m, n), H_{m, n}$ is the complex channel frequency response (CFR) with a variance denoted by $\sigma_{H}^{2}=E\left\{\left|H_{m, n}\right|^{2}\right\}, E\{$.$\} being the mathematical expectation,$ and $\Omega$ is the set frequency-time positions surrounding $(m, n)$. The channel frequency response $H_{m, n}$ is defined by:

$$
H_{m, n}=\sum_{l=0}^{L_{c}-1} h_{l, n} e^{-2 j \pi \frac{m}{M-1} \tau_{l}},
$$

where $L_{c}$ is the number of paths, $h_{l, n}$ the complex gain of the $l^{\text {th }}$ path and $\tau_{l}$ the corresponding delay. In this paper, the channel $\left|H_{m, n}\right|$ is supposed to have a Rayleigh distribution. $w_{m, n}$ and $I_{m, n}$ are the complex additive non-white Gaussian noise with a variance $\sigma^{2}$ and the interference term with a variance $\sigma_{I}^{2}$ respectively. The term $j<g>_{p, q}^{m, n}$ is called "intrinsic interference" and is defined as the scalar product

$$
\sum_{i} g_{m, n}[i] g_{p, q}^{*}[i] d t=\left\{\begin{array}{ll}
1, & \text { if }(p, q)=(m, n) \\
j<g>_{p, q}^{m, n}, & \text { else }
\end{array} .\right.
$$

where ${ }^{*}$ denotes the conjugate of the prototype filter $g_{m, n}[i]$, which can be expressed for any frequency-time position $(m, n)$ as

$$
g_{m, n}[i]=g\left(i-n \frac{M}{2}\right) e^{\frac{2 j \pi m}{M}\left(i-\frac{L_{f}-1}{2}\right)} e^{j \phi_{m, n}}
$$

where the phase term is $\phi_{m, n}=(\pi / 2)(m+n)+m n \pi$ as defined in [12]. The filter $g_{m, n}$ has a length $L_{f}=K M$, where $K$ is the overlapping factor and $M$ is the total number of subcarriers. In this paper, the Bellanger's filter defined in $[11]$ is considered. The frequency response $G[f]$ of this filter has the following expression

$$
G[f]=\sum_{k=-(K-1)}^{K-1} G_{k} \frac{\sin \left(\pi\left(f-\frac{k}{L_{f}}\right) L_{f}\right)}{L_{f} \pi\left(f-\frac{k}{L_{f}}\right)},
$$

where $G_{0}=1, G_{1}=0.971960, G_{2}=1 / \sqrt{2}$, and $G_{3}=$ 0.235147 according to [11], and $G_{k}=G_{-k}$. The reader can refer to [11] for more details on the general way of computing $G_{k}$ for any $K$ value.

The intrinsic interference values $j<g>_{p, q}^{m, n}$ corresponding to Bellanger's filter with $K=4$ are given for instance in [11] and recalled in Fig. 1-(b). Note that, as assessed in [5], these gains correspond to even $m$ values and must be multiplied by -1 for odd $m$ values. Thus, even in absence of the multipath channel, there will be some intercarrier and intersymbol complex interferences from all the surrounding time-frequency positions $(p, q)$ that belongs to the set $\Omega$ (see Fig. 1-(b)). For a sake of clarity, we also define $\Omega_{0}$ as the set of positions $(p, q)$ that are adjacent to $(m, n)$, namely $\Omega_{0}=\{(p, q) \mid p \in\{m-1, m, m+1\}, q \in$ $\{n-1, n, m+1\},(p, q) \neq(m, n)\}$ and the set $\Omega^{\prime}=\Omega \backslash \Omega_{0}$. For a better understanding, $\Omega_{0}$ and $\Omega^{\prime}$ are pointed out in Fig. 1-(b) with the green area delimited by the solid line, and by red area delimited by the dotted line respectively.

As a matter of fact, the pulse shape $g_{m, n}$ is orthogonal in the real field which allows the receiver to perfectly recover the real OQAM symbols when $H_{m, n}=1$ since the intrinsic interference $j<g>_{p, q}^{m, n}$ is purely imaginary. However, it can be seen in (1) that the channel frequency response (CFR) $H_{p, q}$ induces some complex distortions from the surrounding positions $(p, q)$ (see Fig. 2). As a consequence, the channel has to be estimated and corrected in order to recover the real OQAM symbols $C_{m, n}$. More details regarding the OFDM/OQAM modulation scheme are provided in [1], [5].

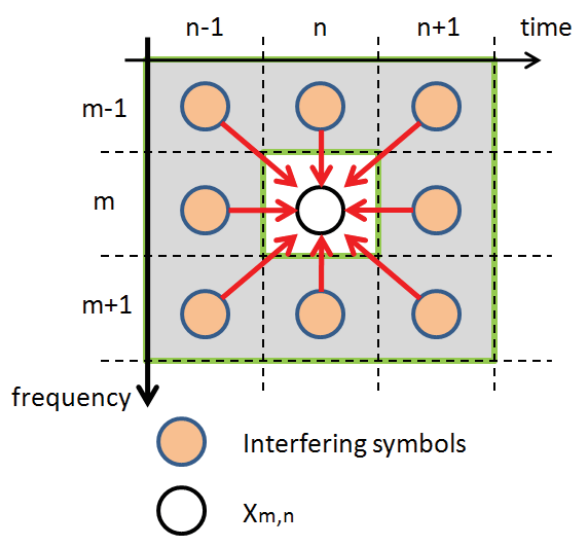

Fig. 2. Interferences generated by the subcarriers and symbols around $X_{m, n}$. 


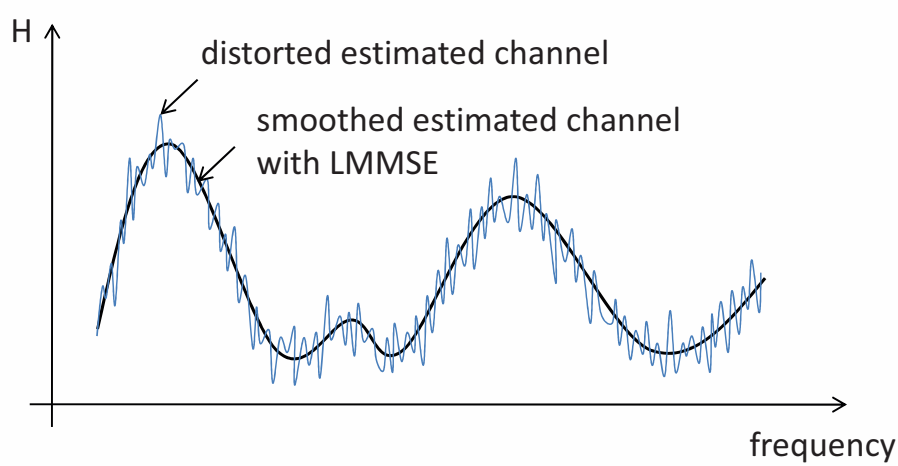

Fig. 3. Illustration of the spectral smoothing effect of LMMSE.

It must be emphasized that if the channel is flat (i.e. $\left.H_{m, n}=1, \forall(m, n)\right)$, then $I_{m, n}$ is purely imaginary, and therefore the symbols are simply detected by: $\hat{X}_{m, n}=\operatorname{Re}\left\{Y_{m, n}\right\}$, where $\operatorname{Re}\{$.$\} is the real part function. In$ the case where $H_{m, n} \neq 1$, the challenge is to estimate and remove the frequency selective channel. In order to achieve this, it has been proposed in [6] to use in OFDM/OQAM the LMMSE channel estimation method, which has been originally presented for OFDM systems in [7].

In order to adapt the LMMSE estimator to OFDM/OQAM scheme, the channel frequency response is assumed to be constant at the frequency and time positions surrounding the position $(m, n)$ [6]. This leads to the approximation $H_{p, q} \approx$ $H_{m, n}$, where $p \in\{m-1, m, m+1\}$ and $q \in\{n-1, n, n+1\}$. Thus, the $M \times 1$ vector of the LMMSE channel estimate can be expressed as

$$
\hat{\mathbf{H}}_{n}^{L M M S E}=\underbrace{\mathbf{R}_{h h}\left(\mathbf{R}_{h h}+\mathbf{R}_{w w}\left(\mathbf{X}_{n} \mathbf{X}_{n}^{H}\right)^{-1}\right)^{-1}}_{\mathbf{K}} \hat{\mathbf{H}}_{n},
$$

where $\hat{\mathbf{H}}_{n}$ is the $M \times 1$ vector of the least square (LS)-based estimate of the channel, which has been obtained thanks to a preamble composed of pilots (see [5] and references therein for details on LS-based estimator in OFDM/OQAM). The $M \times 1$ vector $\mathbf{X}_{n}$ contains the pilot tones used for estimating $\hat{\mathbf{H}}_{n}$. $\mathbf{R}_{h h}$ is the channel covariance matrix defined as $\mathbf{R}_{h h}=E\left\{\mathbf{H}_{n} \mathbf{H}_{n}^{H}\right\}$, where $\mathbf{H}_{n}$ is the $M \times 1$ vector of the channel frequency response, $E\{$.$\} is the mathematical$ expectation, and (. $)^{H}$ the Hermitian transpose. $\mathbf{R}_{w w}$ is the $M \times M$ noise covariance matrix. It must be emphasized that $\mathbf{R}_{w w}$ is a circulant matrix in OFDM/OQAM due to the overlapping prototype filter, whereas it is diagonal in OFDM.

The LMMSE estimator allows to smooth the distortions in the estimated CFR as illustrated in Fig. 3. It has been analytically assessed in [10] that the intrinsic interference in OFDM/OQAM, in addition to the Gaussian noise, is a source of distortion which affects the channel estimation performance. As a consequence, the spectral smoothing achieved by LMMSE appears to be very appropriate in OFDM/OQAM modulation.

For sake of simplicity, it is assumed that the pilots are equi-powered, so that $\mathbf{X}_{n} \mathbf{X}_{n}^{H}=\alpha \mathbf{I}$, where $\alpha \in \mathbb{R}_{+}^{*}$, and $\mathbf{I}$ is the $M \times M$ identity matrix. It can be noted in (6) that the computation of $\mathbf{K}$ in (6) requires both matrix inversion and product, namely a complexity of order $\mathcal{O}\left(M^{3}\right)$ scalar multiplications. Furthermore, the channel covariance matrix is usually unknown at the receiver side. In the following, two LMMSE approximations, which allow to reduce the complexity of the estimator without prior knowledge of $\mathbf{R}_{h h}$, are presented.

\section{Proposed Approximations FOR ApProximating LMMSE IN OFDM/OQAM}

This section deals with two proposed LMMSE-based channel estimators for OFDM/OQAM modulation scheme. Widely studied for OFDM systems as in [8] and [7], the LMMSE estimator suffers from two drawbacks: i) its complexity, and ii) the need of the second order moment of the channel and the noise (see (6)). However, one can benefit from the nature of the matrices $\mathbf{R}_{h h}$ and $\mathbf{R}_{w w}$ in order to reduce the complexity of LMMSE. The two LMMSE-based techniques are referred as "Approximation-1" with proposed one as "Approximation2'. Note referred Approximation 1 approach, has been deeply analyzed by the authors in [6].

\section{A. Approximation 1}

The principle is to approximate the circulant matrix $\mathbf{R}_{w w}$ by the diagonal matrix $\sigma^{2} \mathbf{I}$, where $\sigma^{2}$ is the noise variance. Thanks to this approximation, (6) becomes similar to the formulation of LMMSE in OFDM, and the low rank approximation (LRA) method [7] can be applied to reduce the complexity of LMMSE in OFDM/OQAM, such as in [6]. The basic principle of the LRA is to substitute the unknown matrix $\mathbf{R}_{h h}$ in (6) by a matrix whose elements are perfectly known at the receiver. Thus, the advantages of LRA are twofold: it allows to reduce the complexity of LMMSE (see details hereafter), and avoids the prior knowledge of the channel covariance matrix $\mathbf{R}_{h h}$ at the receiver. The LRA-LMMSE estimator can be expressed as

$$
\hat{\mathbf{H}}_{n, 1}^{L M M S E}=\underbrace{\tilde{\mathbf{R}}_{h h 1}\left(\tilde{\mathbf{R}}_{h h 1}+\frac{\sigma^{2}}{\alpha} \mathbf{I}\right)^{-1}}_{\tilde{\mathbf{K}}_{1}} \hat{\mathbf{H}}_{n},
$$

where $\tilde{\mathbf{R}}_{h h 1}$ is the known low-rank Hermitian covariance matrix which replaces the unknown channel covariance matrix $\mathbf{R}_{h h}$. A constant eigenvalues profile is usually considered for $\tilde{\mathbf{R}}_{h h 1}$ [7]. Therefore, the elements $\left(\tilde{\mathbf{R}}_{h h 1}\right)_{u, v}$ (at the $u$-th row, $v$-th column) of the matrix $\tilde{\mathbf{R}}_{h h 1}$ are expressed by

$$
\left(\tilde{\mathbf{R}}_{h h 1}\right)_{u, v}=\frac{1-e^{-2 j \pi L(u-v) / M}}{2 j \pi L(u-v) / M},
$$

where $L$ is the length of the constant eigenvalues profile ${ }^{1}$. The matrix $\tilde{\mathbf{R}}_{h h 1}$ is diagonalizable in an orthonormal basis

\footnotetext{
${ }^{1}$ This eigenvalues profile correspond to the power delay profile of the channel
} 
so that $\tilde{\mathbf{R}}_{h h 1}=\tilde{\mathbf{U}} \tilde{\boldsymbol{\Delta}}_{R} \tilde{\mathbf{U}}^{H}$. According to the used constant profile, the diagonal elements of $\tilde{\boldsymbol{\Delta}}_{R}$ are $\tilde{\lambda}_{m}=1 / L$, if $0 \leq$ $m \leq L-1$, and $\tilde{\lambda}_{m}=0$, if $L \leq m \leq M-1$. It can be easily demonstrated that $\tilde{\mathbf{R}}_{h h 1}$ and $\left(\tilde{\mathbf{R}}_{h h 1}+\frac{\sigma^{2}}{\alpha} \mathbf{I}\right)^{-1}$ are diagonalizable in the same basis. As a consequence, (7) can be simply rewritten as $\hat{\mathbf{H}}_{n, 1}^{L M S E}=\tilde{\mathbf{U}} \tilde{\boldsymbol{\Delta}}_{K_{1}} \tilde{\mathbf{U}}^{H} \hat{\mathbf{H}}_{n}$, where $\tilde{\boldsymbol{\Delta}}_{K_{1}}$ is the eigen decomposition of $\tilde{\mathbf{K}}_{1}$ with diagonal entries $\tilde{\mu}_{m 1}$ so that

$$
\tilde{\mu}_{m 1}=\left\{\begin{array}{ll}
\frac{\tilde{\lambda}_{m}}{\tilde{\lambda}_{m}+\frac{\sigma^{2}}{\alpha}} & \text { if } m=0,1, . ., L-1 \\
0 & \text { if } m=L, . ., M-1
\end{array} .\right.
$$

\section{B. Approximation 2}

We approximate the Hermitian matrix $\mathbf{R}_{h h}$ by a circulant matrix denoted by $\tilde{\mathbf{R}}_{h h 2}$. Thus, the LMMSE estimation in (6) can be rewritten as

$$
\hat{\mathbf{H}}_{n, 2}^{L M M S E}=\underbrace{\tilde{\mathbf{R}}_{h h 2}\left(\tilde{\mathbf{R}}_{h h 2}+\frac{1}{\alpha} \mathbf{R}_{w w}\right)^{-1}}_{\tilde{\mathbf{K}}_{2}} \hat{\mathbf{H}}_{n},
$$

where $\tilde{\mathbf{R}}_{h h 2}$ is defined as $\mathcal{F} \tilde{\boldsymbol{\Delta}}_{R} \mathcal{F}^{H}$. The diagonal matrix $\tilde{\boldsymbol{\Delta}}_{R}$ remains the same as previously, and $\mathcal{F}$ is the FFT matrix whose entries $(\mathcal{F})_{u, v}$ are equal to $\exp \left(-2 j \pi \frac{u v}{M}\right)$, for any $u, v=0,1, . ., M-1$. Since any circulant matrix is diagonalizable in the Fourier basis, then (10) can be rewritten $\hat{\mathbf{H}}_{n, 2}^{L M M S}=\mathcal{F} \tilde{\boldsymbol{\Delta}}_{K_{2}} \mathcal{F}^{H} \hat{\mathbf{H}}_{n}$, where $\tilde{\boldsymbol{\Delta}}_{K_{2}}$ is the eigen decomposition of $\tilde{\mathbf{K}}_{2}$ with diagonal entries $\tilde{\mu}_{m 1}$ so that

$$
\tilde{\mu}_{m 1}=\left\{\begin{array}{ll}
\frac{\tilde{\lambda}_{m}}{\tilde{\lambda}_{m}+\frac{\tilde{\sigma}_{m}^{2}}{\alpha}} & \text { if } m=0,1, . ., L-1 \\
0 & \text { if } m=L, . ., M-1
\end{array},\right.
$$

where $\tilde{\sigma}_{m}^{2}$ are the eigenvalues of $\mathbf{R}_{w w}$. Unlike in (9), these eigenvalues are not constant for any $m$ as $\mathbf{R}_{w w}$ is not diagonal. However, it can be noticed that the shape of $\tilde{\sigma}_{m}^{2}$ only depends on $\sigma^{2}$ and on the used prototype filter.

The complexity of the different methods, i.e. LMMSE in (6), Approximation-1 in (7), and Approximation-2 in (10) are summarized in Table I. In can be noted that the complexity of Approximation-2 is highly reduced in comparison with LMMSE and Approximation-1, since it uses the FFT matrix for the eigen decomposition of $\tilde{\mathbf{K}}_{2}$.

\section{TABLE I}

COMPLEXITY COMPARISON OF THE LMMSE-BASED ESTIMATORS

\begin{tabular}{|l|l|}
\hline Estimator & Complexity \\
\hline LMMSE (2) & $\mathcal{O}\left(M^{3}\right)$ \\
\hline Approximation-1 (3) & $\mathcal{O}\left(M^{2}\right)$ \\
\hline Approximation-2 (6) & $\mathcal{O}\left(M \log _{2}(M)\right)$ \\
\hline
\end{tabular}

\section{Simulations Results}

The used simulations parameters are listed hereafter: the symbols are composed of 256 subcarriers of $15 \mathrm{kHz}$ each, modulated with a 4-QAM or 16-QAM constellation.
The PHYDYAS prototype filter with overlapping factor $K=4$ (see [5], [6] for more details) is considered. The noise variance $\sigma^{2}$ is supposed to be known at the receiver. The used channel model is the typical urban (20 paths and maximum delay $\tau_{\max }=2.14 \mu \mathrm{s}$ ), and BER performances are presented without channel coding. The pilot pattern used in OFDM/OQAM is the interference cancellation method (ICM) (see [5], [6] for more details).

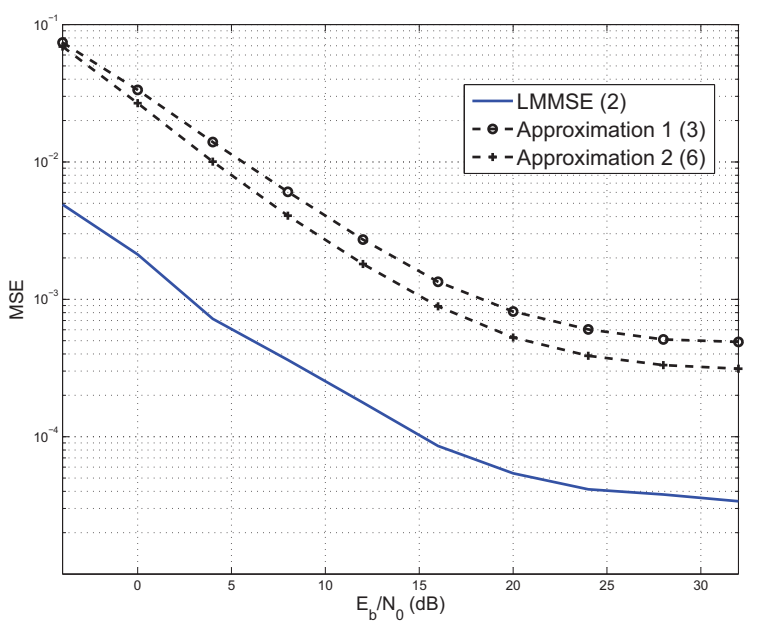

Fig. 4. MSE versus $E_{b} / N_{0}(\mathrm{~dB})$. Performance comparison between LMMSE in (6), Approximation-1 in (7), and Approximation-2 in (10).

Fig. 4 shows the MSE performances versus $E_{b} / N_{0}(\mathrm{~dB})$ of proposed Approximation-1 and Approximation-2. The LMMSE in (6) using the exact covariance matrix is plotted as well. It can be observed that Approximation-2 achieves a lower MSE than Approximation- 1 for any considered $E_{b} / N_{0}$ value. It is worth noticing that the performance of LMMSE reaches a lower bound, an analytical value of which has been proposed in [10]. We deduce that Approximation-2 fits better the OFDM/OQAM modulation scheme than Approximation-1, which is an adaptation of a technique originally proposed for OFDM. It can be also noted than LMMSE in (6) outperforms both proposed methods. However, it's important to highlight that obtaining the LMMSE such as in (6) is very complex for real implementation, as we need to known the value of the matrix $\mathbf{R}_{h h}$ which is difficult to obtain.

Fig. 5 shows the BER performances versus $E_{b} / N_{0}(\mathrm{~dB})$ using LMMSE in (6) and proposed approximations in (7) and (10) for 4-QAM and 16-QAM constellations. The perfect estimation is plotted as a reference. The BER curves have been obtained after a one-tap zero-forcing equalization. It can be highlighted that all the LMMSE-based simplified estimation methods achieve similar performance for 4-QAM and 16QAM, in the considered $E_{b} / N_{0}$ range, and have similar performances to exact LMMSE in $E_{b} / N_{0}$ range from $-4 \mathrm{~dB}$ to $20 \mathrm{~dB}$. Note that the huge difference in the MSE performance does not appears on the BER results. This is mainly due to the effect of the equalization and the detection stages. Therefore, 


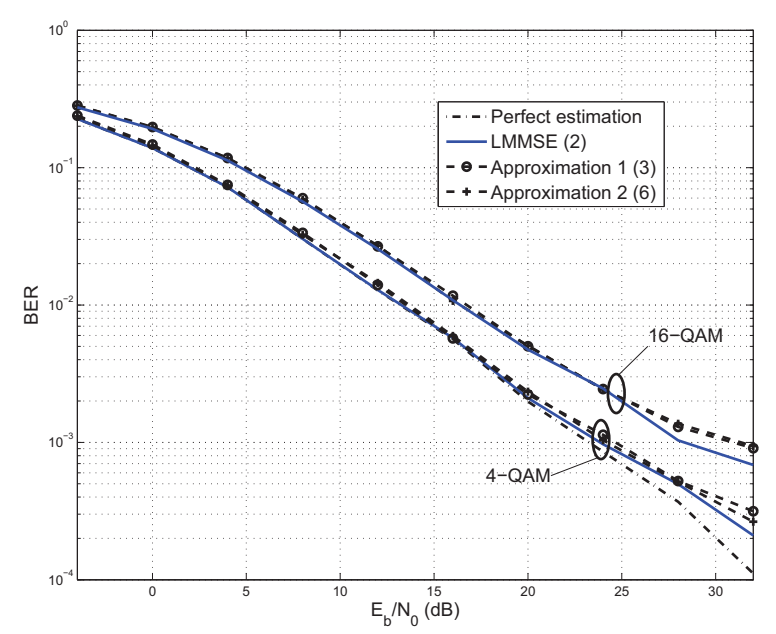

Fig. 5. BER versus $E_{b} / N_{0}(\mathrm{~dB})$. Performance comparison between perfect estimation LMMSE (6), Approximation-1 in (7), and Approximation-2 in (10).

both approximations are valid for LMMSE in terms of BER performance, but it is worth noticing that Approximation-2 is less complex than Approximation-1, see complexity rates in Table I.

\section{CONCLUSION}

In this paper, we have compared two solutions for the complexity reduction of LMMSE in OFDM/OQAM. A first approximation (labeled as Approximation-1) was originally designed for the OFDM modulation [7], and was used in OFDM/OQAM in [6]. The prpoposed second approximation (labeled as Approximation-2) in this paper is more adapted to OFDM/OQAM, since the circulant feature of the noise covariance matrix is taken into account in the LMMSE approximation. The results revealed that both proposed approximations have very similar performances, but Approximation-1 is more complex than Approximation-2. As a consequence, we can conclude that the proposed Approximation- 2 is a very good candidate for low-complexity LMMSE estimation in OFDM/OQAM.

\section{ACKNOWLEDGMENT}

This work is supported by the project PROFIL (Evolution of the wideband PROfessional Mobile Radio based on the FILter Bank MultiCarrier modulation) funded by the French national research agency (ANR) with grant agreement code: ANR-13INFR-0007-03.

\section{REFERENCES}

[1] B. Farhang-Boroujeny, "OFDM Versus Filter Bank Multicarrier," IEEE Signal Processing Magazine, vol. 28, no. 3, pp. 92 - 112, May 2011.

[2] B. L. Floch, M. Alard, and C. Berrou, "Coded Orthogonal Frequency Division Multiplex," Proceedings of the IEEE, vol. 83, no. 6, pp. 982996, June 1995.

[3] J.-P. Javaudin, D. Lacroix, and A. Rouxel, "Pilot-Aided Channel Estimation for OFDM/OQAM," in proc. of VTC'03 Spring, vol. 3, Jeju, Korea, April 2003, pp. 1581 - 1585.
[4] C. Lélé, J.-P. Javaudin, R. Legouable, A. Skrzypczak, and P. Siohan, "Channel estimation methods for preamble-based OFDM/OQAM modulations," European Transactions on Telecommunications, vol. 19, no. 7, pp. $741-750$, November 2008.

[5] E. Kofidis, D. Katselis, A.Rontogiannis, and S. Theodoridis, "Preamblebased channel estimation in OFDM/OQAM systems: A review," Signal Processing, Elsevier, vol. 93, pp. 2038 - 2054, January 2013.

[6] V. Savaux, F. Bader, and Y. Louët, "A Joint MMSE Channel and Noise Variance Estimation for OFDM/OQAM Modulation," IEEE Transactions on Communications, vol. 63, no. 11, pp. 4254 - 4266, November 2015.

[7] O. Edfors, M. Sandell, J.-J. van de Beek, S. K. Wilson, and P. O. Börjesson, "OFDM Channel Estimation by Singular Value Decomposition," IEEE Trans. on Communications, vol. 46, no. 7, pp. 931 - 939, July 1998.

[8] P. Hoeher, S. Kaiser, and P. Robertson, "Two-Dimensional Pilot-SymbolAided Channel Estimation by Wiener Filtering," in ICASSP, vol. 3, Munich, April 1997, pp. 1845 - 1848.

[9] E. Kofidis and D. Katselis, "Improved Interference Approximation Method for Preamble-Based Channel Estimation in FBMC/OQAM," in European Signal Processing Conference (EUSIPCO'11), Barcelona, Spain., 2011, p. 16031607.

[10] V. Savaux and F. Bader, " Mean Square Error Analysis and LMMSE Application for Preamble-Based Channel Estimation in OFDM/OQAM Systems," IET Communications Journal, vol. 9, no. 14, pp. 1763-1773, 2015.

[11] M. Bellanger, "Specification and Design of a Prototype Filter for Filter Bank Based Multicarrier Transmission,' in proc. of ICASSP'01, vol. 4, Salt Lake City, UT, May 2001, pp. 2417 - 2420.

[12] P. Siohan, C. Siclet, and N. Lacaille, "Analysis and Design of OFDM/OQAM Systems Based on Filterbank Theory," IEEE Transactions on Signal Processing, vol. 50, no. 05, pp. 1170 - 1183, May 2002. 